\title{
LOS SISTEMAS GANADEROS CON CRIOLLO LECHERO TROPICAL (REYNA) EN COSTA RICA ${ }^{1}$
}

\author{
Julio Vilaboa-Arroniz ${ }^{2}$, Olman Quirós-Madrigal ${ }^{3}$, Pablo Díaz-Rivera ${ }^{2}$, Rodolfo WingChing-Jones ${ }^{4}$, \\ Natalie Brower-Keating ${ }^{5}$, Pedro Zetina-Córdoba ${ }^{6}$
}

\section{RESUMEN}

Los sistemas ganaderos con criollo lechero tropical (Reyna) en Costa Rica. El objetivo del presente trabajo fue identificar los componentes tecnológicos utilizados en los ranchos ganaderos con bovinos Criollo Lechero Tropical (CLT) Reyna y las características socioeconómicas de los productores que utilizan esta raza. Este estudio se realizó de mayo a julio de 2011 en las provincias de Cartago y Guanacaste, Costa Rica. Se entrevistó a los administradores de los módulos lecheros de la Estación Experimental Alfredo Volio Mata (EEAVM) de la Universidad de Costa Rica y del Centro Agronómico Tropical de Investigación y Enseñanza (CATIE) además de cinco productores independientes. Se analizaron variables sociales, tecnológicas y comerciales además de la percepción sobre el uso de esta raza. La información obtenida se analizó mediante estadística descriptiva y análisis de cluster utilizando el programa SAS versión 8.0. Se encontraron cuatro grupos: denominados G1: productores de Guanacaste, G2: EEAVM, G3: productor de Cartago y G4: CATIE. La superficie para estos grupos fue de $60 \pm 31,6 ; 24 ; 57 ; 46$ ha con una carga animal (UA/ha) de $0,6 \pm 0,1 ; 2,6 ; 0,7$ y 4,1 , respectivamente. La raza CLT Reyna se utiliza principalmente para establecer cruces con Bos taurus y/o Bos indicus con la finalidad de obtener adaptabilidad y resistencia al medio. Sin embargo, su utilización y manejo ha ido en detrimento por políticas institucionales, aspectos legales y productivos.

Palabras clave: Razas, cruces, producción de leche, índice tecnológico, unidades de producción.

\begin{abstract}
Livestocks systems with tropical dairy creolle (Reyna) in Costa Rica. The aim of this study was to identify the technology components used in livestock systems with Tropical Dairy Creole (CLT) Reyna and socioeconomic characteristics of farmers who use this race. The study was conducted in the provinces of Cartago and Guanacaste, Costa Rica in May-July 2011. We interviewed managers of dairy modules at Experiment Station Alfredo Volio Mata (EEAVM) at the University of Costa Rica and the Tropical Agricultural Research and Education (CATIE) plus five independent producers. Variables analyzed were social, technological and commercial awareness as well as the use of this breed. The data was analyzed by descriptive statistics and cluster analysis using SAS version 8.0. There were four groups called G1: Guanacaste producers, G2: EEAVM, G3: Cartago producer and G4: CATIE. The surface for these groups was $60 \pm 31.6,24,57$ and 46 hectares with a united animal per hectare (AU ha-1) $0.6 \pm 0.1,2.6,0.7$ and 4.1 , respectively. Race Reyna is mainly used to establish Bos taurus and/or Bos indicus crosses in order to obtain adaptability and resistance to the environment. However, its use and management has been undermined by institutional policies, both legal and productive.
\end{abstract}

Key words: Races, crosses, milk production, technology index, production units.

\footnotetext{
1 Recibido: 11 de octubre, 2011. Aceptado: 12 de marzo, 2012. Estancia Corta de Investigación Postdoctoral. Centro de Investigaciones en Economía Agrícola y Desarrollo Agroempresarial (CIEDA). Universidad de Costa Rica (UCR).

2 Colegio de Postgraduados Campus Veracruz. Km. 88,5 Carretera Federal Xalapa-Veracruz. Predio Tepetates. Veracruz, México. juliovilaboa@hotmail.com; pablod@colpos.mx

3 Centros de Investigaciones en Economía Agrícola y Desarrollo Agroempresarial (CIEDA). Universidad de Costa Rica. Ciudad Universitaria Rodrigo Facio. San Pedro, Costa Rica. olman.quiros@ucr.ac.cr

4 Escuela de Zootecnia. Universidad de Costa Rica. Ciudad Universitaria Rodrigo Facio. San Pedro, Costa Rica. rodolfo.wingching@ucr.ac.cr

5 Peace Corps. Costa Rica - Tico 19. natalie.keating@gmail.com

6 Universidad Politécnica de Huatusco. Unidad Académica de Biotecnología y Agroindustrial. Avenida 1 No. 728. Col. Centro. C.P. 94100. Huatusco, Veracruz, México. zecp_mc@hotmail.com
} 


\section{INTRODUCCIÓN}

En Costa Rica, la ganadería bovina es una actividad de importancia en el sector agropecuario, aporta el $75 \%$ del Producto Interno Bruto pecuario (PIB) y $16 \%$ del PIB del sector primario (SEPSA 2002). Se tiene un inventario superior a 38000 unidades productivas (UP) de las cuales el 63\% se enfocan a la producción de carne y $37 \%$ a la producción de leche; de éstas $45 \%$ son sistemas especializados y $55 \%$ doble propósito (DP) (Cámara Nacional de Productores de Leche, 2004). El $80 \%$ de las fincas cuentan con superficies menores a 50 ha; el $39 \%$ de éstas poseen menos de 10 ha mientras que sólo un $9 \%$ de las fincas cuentan con más de 80 ha y concentran el $42 \%$ del hato nacional (Corfoga 2000).

La producción de leche es de 790 millones de litros por año (Cámara Nacional de Productores de Leche 2004). Las principales razas utilizadas son la Holstein, Jersey (95\%) y sus cruces con otras razas Bos taurus o Bos indicus. Las lecherías especializadas se concentran en las regiones centro y norte, mientras que en la zona sur predomina el DP (MAG 2007).

A nivel continente americano, hay poca utilización y desarrollo de razas criollas, aspecto que ha causado la disminución del inventario de éstas (Müller-Hayer y Gelman 1981). La desvalorización de las razas nativas se debe a la incorporación de razas o líneas especializadas en producción de leche. Para el caso de Costa Rica, la importación de razas (Holstein, Jersey y Suizo, principalmente) inició en la década de los 50's en demérito del ganado criollo lechero tropical (MAG, 2007). No obstante, las razas especializadas han requerido de un mayor manejo alimenticio-sanitario para poder adaptarse al trópico (Pariacote 2000).

La raza CLT Reyna también conocida como Criollo Lechero Tropical Criollo o Lechero Centroamericano es una raza formada por bovinos provenientes de Nicaragua, Costa Rica y México (Primo 1992, Santellano et al. 2011). El hato fundador se originó en la zona de Rivas, Nicaragua (por Don Joaquín Reyna) en los años 20's con bovinos procedentes de España que fueron introducidos en el siglo XIV (Rouse 1977, De Alba 2011). Se considera como una raza nativa de Centroamérica especialista en producción de leche a base de pastos naturales, además de presentar adaptación al medio y resistencia tanto al stress generado por el clima tropical como a ecto y endoparásitos (AMCROLET 1998, González et al. 2009).
Según la Asociación Mexicana de Criadores de Romosinuano y Criollo Lechero Tropical (AMCROLET), organismo que agrupa socios de México, Nicaragua y Costa Rica, se tiene un inventario aproximado de 1,675 bovinos. Dicha asociación tiene 88 socios, de los cuales $68 \%$ se consideran como activos y $32 \%$ como no-activos, además que existen otros hatos y productores no registrados (AMCROLET 1998; 2010).

Para el caso de Costa Rica, la AMCROLET (2010) reporta como socios al Instituto Tecnológico de Costa Rica, la Universidad EARHT, el Centro Experimental Alfredo Volio Mata de la Universidad de Costa Rica (EEAVM) y el Centro Agronómico Tropical de Investigación y Enseñanza (CATIE); los dos primeros con ganado Romosinuano y los dos últimos con CLT Reyna. El desarrollo y conservación de esta raza ha estado a cargo principalmente de centros de enseñanza e investigación (De Alba 2007) mediante apoyo internacional (FAO 2004a). Sin embargo, estos esfuerzos no han generado el impacto deseado sobre los ganaderos (Wilkins 1991, De Alba 2007), posiblemente por desconocimiento de la raza o moda en la utilización de razas productoras de leche exóticas.

En Costa Rica la raza CLT Reyna tiene cierta importancia sociocultural; no obstante, se encuentra amenazada por la modificación de los sistemas de producción, además de que se desconoce la población actual y las condiciones de pureza de muchos hatos (Hidalgo et al. 2004). Por su parte, Rubio (2008) menciona que dicha raza tuvo mayor impacto por la exportación de semen por parte del CATIE que por el establecimiento de sistemas de producción con dicha raza.

Los estudios referentes a la raza CLT Reyna se han orientado a selección y mejoramiento genético (Magofke 1964, Deaton 1979); comportamiento productivo y reproductivo (Rosendo y Becerril 2002, Díaz et al. 2005, Martínez et al. 2006), respuesta a factores ambientales (Hernández et al. 2007); resistencia a ectoparásitos (González et al. 2009), características de la leche y sus derivados (Estrella et al. 2004, Guerrero et al. 2004) así como su comparación con otras razas lecheras o doble propósito (Casas y Tewolde 2001, Cervantes et al. 2006, Martínez et al. 2006); pero, se carece de estudios orientados a las áreas técnico-productiva y socioeconómica, las cuales están más relacionadas con los productores.

El objetivo del presente trabajo fue identificar los componentes tecnológicos utilizados en los ranchos 
ganaderos costarricenses que utilizan la raza CLT Reyna y determinar las características socioeconómicas de los productores que utilizan esta raza.

\section{MATERIALES Y MÉTODOS}

Costa Rica, ubicado en Centroamérica, tiene una superficie de $50900 \mathrm{~km}^{2}$; se localiza geográficamente en las coordenadas $9^{\circ} 56^{\prime} 0^{\prime \prime} \mathrm{LN}, 84^{\circ}$ 5' 0" LO. Limita al norte con Nicaragua, al sureste con Panamá, al este con el mar Caribe y al oeste y suroeste con el Océano Pacífico. Se divide socioeconómicamente en seis regiones y se subdivide en siete provincias, 81 cantones y 463 distritos.

Las principales regiones lecheras son Huetar Norte, Central y Chorotega que en su conjunto representan más del $72 \%$ de la producción nacional (Cuadro 1). Estas regiones se caracterizan por sistemas intensivos y la producción se destina al sector industrial. El $28 \%$ restante de la producción nacional se divide entre las regiones Brunca (13\%), Huetar Atlántica (10\%) y Pacífico Central (5\%) donde destacan los sistemas extensivos doble propósito y el volumen de la producción se distribuye entre el sector industrial y el comercio artesanal (Alfaro 2007).

El estudio se realizó en las provincias de Cartago $\left(9^{\circ} 52^{\prime} \mathrm{N}\right.$ y $\left.83^{\circ} 55^{\prime} \mathrm{O}\right)$ y Guanacaste $\left(10^{\circ} 15^{\prime} \mathrm{N}\right.$ y $85^{\circ}$ $\left.41^{\prime} \mathrm{O}\right)$ en los cantones de Cartago, Turrialba, Liberia y Santa Cruz de marzo a junio del 2011. La temperatura media anual en Guanacaste varía entre $26-33^{\circ} \mathrm{C}$ en la parte baja y entre $9-11^{\circ} \mathrm{C}$ en la parte alta con una precipitación media anual entre 1500 y 2000 mm. Por

Cuadro 1. Porcentaje de producción de leche por región y provincia en Costa Rica.

\begin{tabular}{lcc}
\hline Región & Provincia & $\begin{array}{c}\text { Aportación } \\
(\%)\end{array}$ \\
\hline Huerta Norte y Central & Alajuela & 59,3 \\
Central & Cartago & 17,0 \\
Central & San José & 7,4 \\
Chorotega & Guanacaste & 6,9 \\
Huetar Norte, Huetar Atlántica, & & \\
Central & Heredia & 4,7 \\
Resto del país & Otras & 4,7 \\
\hline
\end{tabular}

Fuente: Cámara Nacional de Productores de Leche, 2004. su parte, la provincia de Cartago presenta una precipitación cercana a los $2000 \mathrm{~mm}$ con 128 días con lluvia y un solo mes seco. La temperatura máxima promedio es de $26^{\circ} \mathrm{C}$ y la mínima promedio de $15^{\circ} \mathrm{C}$ (Atlas Cantonal de Costa Rica 1987).

Se entrevistó a personal de la EEAVM (Cartago) y del CATIE (Turrialba) para obtener información referente a productores que utilizan la raza CLT Reyna. Se consultó las fichas de participantes del Diálogo sobre Recursos Genéticos Animales en la Agricultura (Vaughan et al. 2005). Se utilizó un muestreo no probabilístico por cuotas; el criterio de selección fue ser productor que utilice la raza CLT Reyna o cruces con ésta.

Una limitación de la investigación radicó en la falta de un padrón actualizado de productores que utilizan dicha raza; por tanto, se tuvo que enmarcar el estudio con base a la información disponible en las instituciones registradas ante AMCROLET. Se entrevistó a personal de la EEAVM y CATIE además de cinco productores independientes. Las entrevistas se aplicaron de mayo a julio de 2011 .

Para caracterizar a los sistemas ganaderos con ganado CLT Reyna se utilizó la metodología propuesta por Vilaboa y Díaz (2009) modificando el IT para sistemas de producción de leche (Cuadro 2).

Se analizaron veintinueve variables, divididas en aspectos sociales, tecnológicos y de comercialización. Las sociales fueron siete: género, edad, escolaridad, nivel de organización, tipo de mano de obra empleada, administración del rancho, utilización de registros productivos- económicos.

Las variables tecnológicas fueron 16: finalidad zootécnica, propósito de la ganadería, tipo SP, razas utilizadas, conformación del hato, utilización de ordeño, número de vacas en ordeño, litros diarios producidos, tipo y superficie de pastos utilizados, método de pastoreo, forma de conservar el forraje, tipo y épocas de suplementación, tipo de animales suplementados, participación en campañas sanitarias (tuberculosis y brucelosis), principales enfermedades en sus hatos y asistencia técnica.

Las variables comerciales fueron seis: producto a comercializar (leche o queso), forma de venta, destino del producto, precios (leche, queso y ganado), ingresos por venta de leche y por hectárea (ha).

Se diseñó una base de datos en Excel en donde se capturaron los datos obtenidos en campo; posteriormente se clasificaron y depuraron las variables. 
Cuadro 2. Actividades realizadas por los productores en los sistemas de producción de leche con Criollo Lechero Tropical (CLT Reyna) en Costa Rica, consideradas para determinar el índice tecnológico. 2011.

\begin{tabular}{lcc}
\hline Actividad & Rango & Ponderación \\
\hline Registros económicos & $0-1$ & 0,5 \\
Registros productivos & $0-1$ & 0,5 \\
Asesoría técnica & $0-1$ & 1 \\
Sistema de pastoreo & $0-2$ & 0,5 \\
Carga animal (UAha ${ }^{-1}$ ) & $0,5-5,0$ & 1 \\
Tipo de pastos & $0-1$ & 0,5 \\
Suplementación & $0-1$ & 0,5 \\
Desparasitación & $0-1$ & 0,5 \\
Vacunación & $0-1$ & 0,5 \\
Ordeño mecánico & $0-1$ & 0,75 \\
Pesaje de leche & $0-1$ & 0,5 \\
Valor agregado (queso) & $0-1$ & 0,5 \\
Tipo de empadre & $0-3$ & 0,75 \\
Evaluación del semental & $0-1$ & 0,5 \\
\hline
\end{tabular}

Los datos fueron analizados con el paquete estadístico SAS versión 2003 para realizar un análisis descriptivo y posteriormente un análisis cluster.

Se identificaron cuatro grupos de ganaderos, los cuales se denominaron como G1 productores de Guanacaste, G2: EEAVM, G3: productor de Cartago y G4: CATIE.

\section{RESULTADOS Y DISCUSIÓN}

\section{"Entrevistas realizadas"}

\section{Estación Experimental Alfredo Volio Mata $(E E A V M)$}

Se entrevistó, mediante una guía de preguntas, al encargado del módulo lechero de la EEAVM. Según el entrevistado, la raza CLT Reyna comenzó a manejarse en la estación experimental a partir del 2005. Se decidió utilizar dicha raza para probar cruces con Jersey, que también es un Bos taurus, con la finalidad de ofrecer alternativas de producción, considerando tanto las condiciones adversas del entorno, como el clima y del productor (ingresos).

Inicialmente se importó semen (veinticinco fajillas) de Nicaragua del hato de Rivas, ya que en Costa Rica no se contaba con sementales puros de esta raza. Llegaron a manejar hasta 40 bovinos (Jersey x Reyna) con una media de 9 litros por ordeña por vaca. Actualmente manejan 69 bovinos de los cuales sólo seis son cruce $1 / 2$ Jersey x $1 / 2$ Reyna.

Cuando la raza CLT Reyna fue denominada como patrimonio nacional de Nicaragua, se dejó de importar semen debido al incremento en los costos de importación, así como los complejos trámites burocráticos, aspecto que desestimuló el manejo de esta raza en la EEAVM y por consiguiente la adquisición de semen y/o animales. A partir del 2009, los bovinos (cruces) fueron vendidos a productores de la región de Chorotega, principalmente en la provincia de Guanacaste (cantones Liberia y Santa Cruz).

El entrevistado manifestó estar de acuerdo en recomendar el uso de la raza CLT Reyna a los productores, ya que dicha raza tiene potencial de adaptación en zonas secas (del Pacífico), presenta una piel dura que resiste el clima y parásitos externos, y además presenta mansedumbre y puede basar su alimentación sólo en pastos. Dentro de las debilidades mencionó alta consanguinidad en los hatos e importación ilegal (sin registros de procedencia) de bovinos (Cuadro 3).

Centro Agronómico Tropical de Investigación y Educación (CATIE)

Se entrevistó al administrador del módulo lechero del CATIE mediante guía de preguntas. El responsable mencionó que la raza CLT Reyna comenzó a utilizarse a finales de la década de los 50's y principio de los 60`s. Se adquirió dicha raza con fines de investigación y experimentación con cruces con otras razas lecheras con la finalidad de lograr resistencia y adaptación al trópico.

Los primeros sementales (tres) se importaron del hato de Rivas en Nicaragua; posteriormente el CATIE desarrolló su propio semen tanto para uso interno como para venta a nivel nacional e internacional. En el hato se llegaron a manejar hasta ochenta vientres CLT Reyna con un promedio de producción de 15 litros por vaca en dos ordeñas. Actualmente manejan 140 vientres de las cuáles cinco son 3/4 Jersey x 1/4 CLT Reyna. 
Cuadro 3. Principales resultados de las entrevistas realizadas a los productores de los sistemas ganaderos con Criollo Lechero Tropical (CLT Reyna) en Costa Rica. 2011.

\begin{tabular}{|c|c|c|c|c|c|c|c|c|}
\hline $\begin{array}{l}\text { Nombre siste- } \\
\text { ma ganadero }\end{array}$ & $\begin{array}{l}\text { Ubicación } \\
\text { (Provincia) }\end{array}$ & $\begin{array}{l}\text { Periodo } \\
\text { (años) }\end{array}$ & Finalidad & $\begin{array}{l}\text { Origen del } \\
\text { CLT (País) }\end{array}$ & Producto & $\begin{array}{c}\text { Cantidad } \\
\text { adquirida } \\
\left(\mathbf{n}^{\circ}\right) \mathrm{CLT}\end{array}$ & $\begin{array}{l}\text { Inventario } \\
\text { máximo } \\
\left(\mathbf{n}^{\circ}\right) \text { CLT }\end{array}$ & $\begin{array}{l}\text { Inventario } \\
\text { actual }\left(n^{\circ}\right) \\
\text { de CLT }\end{array}$ \\
\hline EEAVM & Cartago & 2005-2009 & Cruces & Nicaragua & Semen & $25(\mathrm{p})$ & 40 & 6 \\
\hline CATIE & Turrialba & 1960-1999 & Investigación & Nicaragua & Bovinos & 3 (b) & 80 & 5 \\
\hline Liberia "A" & Guanacaste & 1986-2011 & Cruces & Nicaragua & Semen & $50(\mathrm{p})$ & 150 & 60 \\
\hline Liberia "B" & Guanacaste & $1981-2011$ & Cruces & Costa Rica & Bovinos & $2(\mathrm{~b})$ & 30 & 30 \\
\hline Santa Cruz "A" & Guanacaste & $2000-2011$ & Cruces & Costa Rica & Bovinos & $5(\mathrm{~b})$ & 18 & 18 \\
\hline Santa Cruz "B" & Guanacaste & 2010-2011 & Cruces & Costa Rica & Bovinos & 1 (b) & 1 & 1 \\
\hline Cartago & Cartago & $2008-2010$ & Cruces & Nicaragua & Semen & $30(\mathrm{p})$ & 0 & 0 \\
\hline
\end{tabular}

Cantidad adquirida: $\mathrm{n}^{\circ}$ de bovinos o pajillas de semen adquiridas. Inventario inicial: $\mathrm{n}^{\circ}$ máximo de bovinos CLT Reyna, puros o cruces con otras razas manejados. Inventario actual: $\mathrm{n}^{\circ}$ de bovinos CLT Reyna, puros o cruces con otras razas, manejados al 2011.

A partir de 1999 se decidió desechar la raza CLT Reyna ya que presentaba periodos muy cortos de lactancia, consanguinidad, mala conformación de ubres (cuartos pequeños o colgantes), mal ángulo del talón que posteriormente originó problemas de movilidad. Además, mencionó que se han desarrollado diversas razas que tienen buena adaptación al trópico.

Actualmente, el módulo se maneja con fines netamente comerciales; enfocado más hacia una producción sostenida durante la vida productiva de las vacas más que la producción por lactancia; por ello realizan cruces con Sawihal, Taurindicus (T40), Holstein Rojo, Gyr Lechero teniendo como base lechera el Jersey.

El ganado CLT Reyna en su mayoría fue destinado al abasto ya que por política institucional los bovinos no podían ser vendidos a productores, por tanto no contaban con información de ellos que manejaran la raza CLT Reyna. El entrevistado mencionó que la producción de leche en el trópico necesita vigor híbrido; recomendaría el uso de la raza CLT Reyna sólo mediante cruces con Jersey pero no hatos puros.

Dentro de los aspectos positivos de la raza, según el entrevistado, son su alta rusticidad, adaptación al medio, resistencia a enfermedades y una alta longevidad (cuentan con una vaca con trece partos) (Cuadro 3).

\section{Productor "A” de Liberia Guanacaste}

Se visitó y entrevistó a un productor en Liberia, Guanacaste. Este tiene aproximadamente veinticinco años de antigüedad y experiencia en el manejo de la raza CLT Reyna. La información sobre la raza la obtuvo del CATIE. En sus inicios adquirió semen (40-50 fajillas) del hato de Rivas en Nicaragua. El objetivo por adquirir la raza CLT Reyna fue establecer cruces con otras razas lecheras con la finalidad de adquirir rusticidad y resistencia al medio ya que los periodos de sequía en su zona de producción son prolongados (siete meses aproximadamente).

Actualmente manejan sesenta bovinos de los cuales el $50 \%$ son CLT puros y $50 \%$ con cruces con Jersey y Pardo Suizo; el entrevistado manifestó que dichos cruces van desde el 25 hasta al 75\% con CLT Reyna y no estar muy de acuerdo en recomendar el uso de dicha raza pues cada productor presenta particularidades específicas; sin embargo, consideró que dicha raza presenta buena producción de leche (12-13/ordeña por vaca), rusticidad al medio, resistencia a ectoparásitos. Dentro de las debilidades de la raza, mencionó que desde la eliminación del hato del CATIE (hace treinta años aproximadamente) se ha perdido información sobre la misma así como disponibilidad de material genético (semen) (Cuadro 3).

\section{Productor "B" de Liberia, Guanacaste}

El segundo productor entrevistado en Liberia, manifestó que ha manejado la raza Reyna por más de treinta años. Este obtuvo información de otros productores y del CATIE. Los primeros ejemplares que 
adquirió venían junto con un lote Doran que había adquirido del CATIE. Según el entrevistado, al observar las aptitudes de la raza (fortaleza y rusticidad) se decidió por adquirir dos toros (1/2 Reyna x $1 / 2$ Jersey) de otros hatos (productor "A" de Liberia). Actualmente, maneja cruces Reyna x Doran, Reyna x Pardo Suizo y Reyna por Jersey en un hato conformado por treinta bovinos. La participación del Reyna en los cruces va desde un 25 hasta un $75 \%$.

En la actualidad cuenta con una producción promedio por vaca de 8 a 10 l/ordeña. Dentro de las fortalezas de la raza, mencionó su rusticidad y adaptabilidad al medio. Por otro lado, manifestó que no hay continuidad en los programas ganaderos, motivo por el cual el CATIE desechó la raza Reyna, además de una falta de selección para el mejoramiento genético y de información a los productores sobre el potencial de la raza CLT Reyna para la producción de leche en el trópico (Cuadro 3).

\section{Productor "A” Santa Cruz, Guanacaste}

El productor entrevistado en Santa Cruz, Guanacaste declaró conocer la raza CLT Reyna desde hace más de veinticinco años pues sus familiares manejaban dicha raza; sin embargo, manifestó que la utilización del CLT Reyna ha ido en detrimento debido a la poca información disponible y difusión entre los productores.

En el año 2000 decidió retomar el uso de la raza CLT Reyna para realizar cruces con otras razas lecheras y doble propósito con la finalidad de obtener resistencia al medio. Dentro de las bondades de la raza CLT Reyna considera la mansedumbre, rusticidad y capacidad de producción de leche mediante pastos de baja calidad. Por otro lado, la debilidad de la raza, según el entrevistado, es referente a la condición corporal de la raza ya que presenta una osamenta gruesa y pronunciada que dificulta el marmoleo (encarnado) y semeja animales de baja condición corporal.

El primer semental lo adquirió en la Universidad de Costa Rica (UCR); las hembras (cuatro) las compró con otro productor de Guanacaste. Actualmente maneja los cruces CLT Reyna x Nerole, CLT Reyna x Brahman y Brahman x Nerole. La participación del CLT Reyna en estos cruces va del 25 al $75 \%$ en un hato conformado por dieciocho vientres con una producción promedio de siete por vaca por ordeña. Finalmente, manifestó que la raza CLT Reyna pudiese ser una alternativa de producción de leche en el trópico ya que existe la necesidad de producir leche a bajo costo (Cuadro 3).

\section{Productor "B" de Santa Cruz, Guanacaste}

Este productor tiene un año de experiencia en el manejo de la raza CLT Reyna. Adquirió un semental puro de la EEAVM de la UCR con para establecer cruces con Brahman y Gyr Lechero. La información sobre la raza la obtuvo de investigadores de la UCR. Dentro de las aptitudes de esta raza, el entrevistado manifestó capacidad de producción de leche y resistencia al calor. Sin embargo, mencionó que los cruces con CLT Reyna deben de hacerse con razas corpulentas ya que ésta, por su aptitud lechera, es de osamenta gruesa de difícil marmoleo. Actualmente maneja cuarenta vientres Gyr Lechero, Brahman y un semental CLT Reyna. Su producción media es de ocho litros por vaca por ordeña (Cuadro 3).

\section{Productor de Cartago}

El productor de Cartago manifestó ser jubilado de la EEAVM donde laboró por 28 años y conoció la raza CLT Reyna. Ya de manera independiente, en 2008, inició con el uso de la raza mediante la compra de treinta pajillas a un productor de Nicaragua como una oportunidad de negocio. En un principio consideró como mercado objetivo a pequeños y medianos productores. Según el entrevistado, el uso de la raza CLT Reyna permitiría a los productores establecer cruces con razas convencionales (Holstein o Jersey) para una producción de leche a bajo costo además de proporcionar vigor híbrido al hato. De igual forma, consideraba que dichos cruces requerían menor infraestructura y componentes tecnológicos para la producción en comparación con las razas especializadas.

El entrevistado decidió el uso del CLT Reyna por una parte, por su experiencia adquirida en la EEAVM; y por la otra, por considerar que el CLT Reyna presenta rusticidad, mansedumbre y adaptación al medio, además de soportar más cambios bruscos en las dietas. Sin embargo, mencionó que la comercialización de semen de ganado CLT Reyna no tuvo el éxito esperado ya que el semen adquirido no contaba con una evaluación o certificación de la calidad del mismo. Además de una baja eficiencia en la inseminación artificial (IA) (cerca del 70\%) ya fuese por la calidad del semen, la conformación corporal de las vacas y/o experiencia de 
la persona que realizaba la IA. Aunado a ello, según el entrevistado, los productores desconocen la raza y las características productivas de la misma por lo que él mismo tenía que realizar labores informativas y de divulgación entre los productores. Debido a ello, en 2010, desechó el negocio de venta de semen y se orientó a la avicultura. Actualmente, cuenta con dos hembras cruces $1 / 2$ CLT Reyna x $1 / 2$ Suizo y machos $1 / 2$ CLT Reyna $x$ 1/2 Simmental en un hato conformado aproximadamente por sesenta bovinos raza Jersey y cruces Jersey x Guersney en diversas proporciones a los cuáles posteriormente le introducirá el cruce con CLT Reyna.

El productor "B" indicó que la debilidad de la raza CLT Reyna es en relación a que presenta una mala conformación de la ubre. Por tanto, considera que el uso de la raza debe ser con Jersey y sólo mediante un programa establecido de cruzamientos; además manifestó que la introducción del CLT Reyna no debe ser en todo el hato sino a una proporción del mismo. Finalmente, el entrevistado mencionó que el término CLT Reyna se debe en honor a Don Joaquín Reyna (hato fundador) ya que antes era conocido como Criollo Lechero Centroamericano; por otro lado, reconoció la importancia de los trabajos realizados por Don Jorge de Alba, como Director del CATIE en la década de los 50`s-60’s con esta raza (Cuadro 3).

\section{Caracterización tecnológica}

La totalidad de los entrevistados fueron hombres con una edad promedio de $52 \pm 8$ años (máximo 62 y mínimo cuarenta años). De los entrevistados uno (14\%) cuenta con estudios de postgrados, cuatro (57\%) con nivel licenciatura y dos (29\%) de bachiller (Cuadro 4). Dos de ellos no pertenecen a ninguna asociación de productores, tres a las cámaras de ganaderas de los cantones de Liberia y Santa Cruz y dos (EEAVM y CATIE) son socios de la Cooperativa de Productores de Leche R.L. Dos Pinos, principal empresa lechera en el país.

El 71\% (5) de las unidades de producción (UP) se dedican a actividades pecuarias; de este porcentaje, un $57 \%$ (cuatro) diversifican la mediante crianza de cabras y conejos (uno), caballos (dos) y avicultura (uno) mientras que los tres productores restantes (43\%) se dedican exclusivamente a la ganadería bovina; el $29 \%$ (dos) cuentan con actividades agrícolas mediante la siembra de caña de azúcar (Sacharum oficinarum).
La superficie agrícola promedio es de $15 \pm 7$ ha mientras que la superficie pecuaria es de $52 \pm 26$ ha. El $100 \%$ de la tenencia de la tierra es propiedad privada. Sólo una de las 7 UP cuenta con 5 ha de riego. El personal de la EEAVM (1) y CATIE (1) se dedican sólo a actividades de docencia e investigación; los demás entrevistados (cinco) manifestaron realizar actividades extra-finca, uno es empleado en el MAG en Liberia y cuatro cuentan con negocios particulares no relacionados con el sector primario.

El $71 \%$ de los entrevistados son administradores de sus propias fincas o responsables de los módulos lecheros de sus respectivas instituciones, mientras $28 \%$ de las UP son manejadas por los hijos u otro familiar. A excepción de la EEAVM y del CATIE, la mano de obra familiar es importante para el desarrollo de la actividad ganadera. En promedio los entrevistados ocupan $4 \pm 3$ personas de mano de obra permanente (siendo mayor en EEAVM) y $3 \pm 2$ de mano de obra eventual. Los productores entrevistados no cuentan con registros productivos (sólo control de nacencias) y económicos; en contraste la EEAMV y CATIE cuentan con registros productivos y en cuanto a los económicos, manejan registros de venta de leche a Dos Pinos.

La experiencia en la actividad ganadera es de $28 \pm 10$

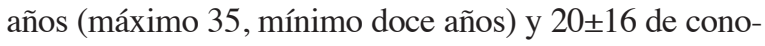
cer la raza CLT Reyna. Los entrevistados manifestaron haber obtenido información sobre el CLT Reyna en Nicaragua (57\%) y Costa Rica (43\%); de éstos el 57\% declaró haber obtenido información en Centros de Investigación y Enseñanza (EEAVM; CATIE y Universidad Nacional de Nicaragua); 29\% de Centro de Enseñanza e Investigación y productores; y, 14\% exclusivamente de productores (Hato de Rivas, Nicaragua). Los principales productos adquiridos fueron semen (57\%), sementales (29\%) y, sementales y vientres (14\%) (Figura 1). Para la EEAVM y CATIE el propósito de incursionar con la raza CLT Reyna fue investigación mientras que para los productores fue el establecimiento de cruces.

Se tiene como base lechera a las razas Jersey y Holstein con diversos cruces. En cuanto al uso de la raza CLT, en la EEAVM y el CATIE se han establecido cruces Jersey x CLT Reyna; por su parte, los productores de Liberia han utilizado Jersey x CLT Reyna, Doran x CLT Reyna, Pardo Suizo x CLT Reyna; en Santa Cruz, el productor, ha utilizado Reina x Nerole, CLT Reyna x Brahman, Brahman x Nerole y CLT Reyna x Brahman x Gyr Lechero y Cartago se han utilizado CLT Reyna x Pardo Suizo, CLT Reyna x Jersey. 


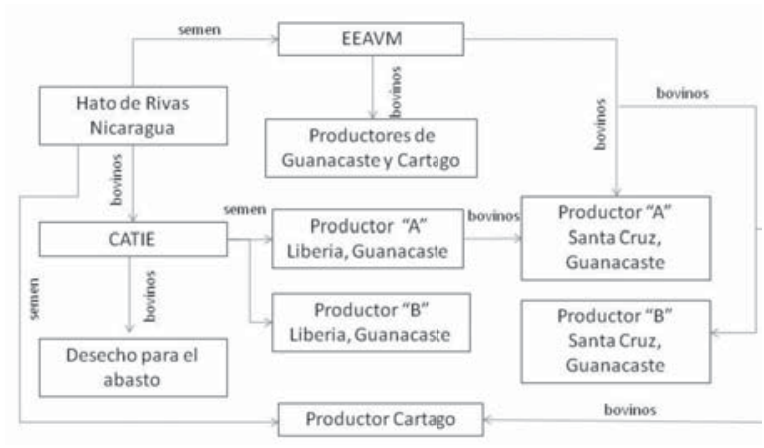

Figura 1. Adquisición y distribución de la raza Criollo Lechero Tropical (Reyna) en los Sistemas Ganaderos de Costa Rica. 2011.

La participación de la raza CLT Reyna es dichos cruces va desde $1 / 4$ hasta $1 / 2$ de sangre (43\%), de $1 / 2$ a $3 / 4$ (43\%) y de $3 / 4$ a $100 \%$ puro (14\%). Es de importancia resaltar que la raza CLT Reyna, en su mayoría, se maneja mediante cruces con diversas razas en las UP visitadas. Sólo uno productor contó con bovinos $100 \%$ puros.

La estructura del hato está conformada por sementales $(1 \%)$, vacas $(57 \%)$, novillonas $(26 \%)$, becerras (13\%) y becerros (3\%). El ganado CLT Reyna sólo conforma el $15 \%$ (14\% vacas en sus diversos cruces, $1 \%$ machos) del total de los hatos y el $24 \%$ de los vientres totales. La mayor cantidad de bovinos CLT Reyna ya sea puros o cruces se encuentran en Liberia y la menor cantidad en el CATIE.

La totalidad de las UP cuentan con ordeñas mecánicas; el ordeño se realiza dos veces (57\%) y una por día (43\%). El pastoreo racional intensivo es característico en la EEAVM y CATIE mientras que los productores utilizan el pastoreo rotacional mediante la utilización de dos o más potreros. Las praderas presentan combinación de pastos inducidos (59\%) y nativos (41\%). Todos ofrecen algún tipo de suplementación al ganado siendo característicos la mineral (14\%) y la mineral-energético-proteica mediante productos comerciales (86\%). La suplementación es proporcionada a todos los animales del hato (42\%), vacas en producción $(29 \%)$ y animales con baja condición corporal (29\%) durante todo el año (57\%) y en periodos de tres a cuatro meses $(43 \%)$.

En cuanto a sanidad, la totalidad de los productores, participan en campañas zoosanitarias (tuberculosis y brucelosis). El 71\% (cinco) de los productores reciben asesoría técnica de Médicos Veterinarios de las provincias donde se ubican las UP mientras que dos productores (29\%) por no ocupar este servicio ni cuenta con calendarios de vacunación y desparasitación. Dichas prácticas se realizan una $(14 \%)$, dos $(43 \%)$ y tres a cuatro veces por año $(29 \%)$ para vacunación y una (29\%) a dos veces (57\%) por año para el caso de desparasitación.

Las principales enfermedades del hato, reportadas por los entrevistados, fueron anaplasmosis, piroplasmosis y fiebre de leche (43\%); mastitis (14\%), septicemia (14\%); el 29\% restante no reportó enfermedades en sus hatos. Por su parte, los principales problemas reproductivos mencionados fueron infecciones uterinas, bajo porcentaje de preñez y retención de becerros al parto.

El tipo de empadre es IA (29\%), monta natural (43\%) y la combinación de ambos (28\%). La detección de calores y gestación se realizan en dos de las siete UP, sin embargo, en ninguna se realiza evaluación del semen o del semental.

La EEAVM y el CATIE se enfocan a la venta de leche a la Cooperativa Dos Pinos; los productores se dedican a la producción y venta de queso; de estos cuatro venden directamente al público y uno a mayorista. El precio por litro de leche varía entre $ф 260-285$ (USD 0,5-0,6) mientras que el precio de venta por $\mathrm{kg}$ de queso es de $\phi 1700$ (USD 3) a mayorista y de entre 2500 a $ф 3000$ (USD 5-6).

Las vacas, al finalizar su vida productiva, son vendidas para el abasto con un peso promedio de 450 $\mathrm{kg}$ con precios basados en la conformación corporal de la misma, los cuales van desde los $\varnothing 40000$ (USD 80) hasta los $₫ 200000$ (USD 400); los becerros son desechados del hato a los pocos días de nacido con un peso promedio de $35-40 \mathrm{~kg}$; éstos son adquiridos por diversos engordadores a puerta de corral quienes engordan y posteriormente vende en las subastas ganaderas.

\section{Caracterización socioeconómica}

Mediante el análisis de conglomerados (cluster) se identificaron cuatro grupos de ganaderos, denominados: G1, G2, G3 y G4 (P<0,05) (Figura 2).

El G1 se conformó exclusivamente por productores de la provincia de Guanacaste, cuenta con edad promedio de $57 \pm 5$ años, presenta escolaridad de 19 $\pm 2,5$ (nivel de licenciatura). Los años de experiencia del G1 en la actividad ganadera es $33 \pm 4$ años 


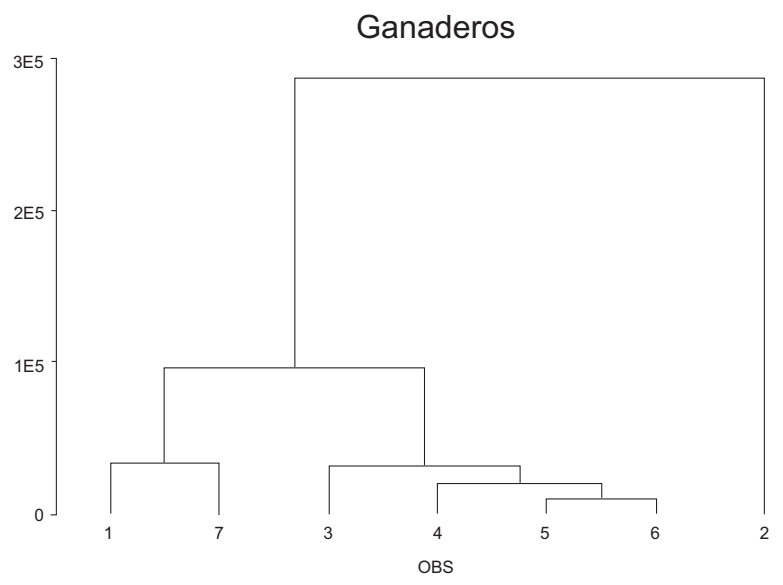

Figura 2. Caracterización socioeconómica y tecnológica de los productores y sistemas ganaderos con Criollo Lechero Tropical (Reyna) en las provincias de Guanacaste y Cartago, Costa Rica. 2011.

(37 máximo y mínimo once año). La superficie es de 60ะ31,6 ha (máximo 100 y mínimo 30 ha). Las unidades animales (UA) son 34,6 $\pm 15,2$ con una carga de 0,6 $\pm 0,1$ UAha $^{-1}$ (máximo 0,8 y mínimo 0,4 $\mathrm{UAha}^{-1}$ ).

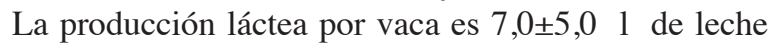
con una producción máxima de 121 . El ingreso por hectárea fue de USD \$297ะ293,75 siendo el máximo de USD\$696. El índice tecnológico fue de cinco (máximo siete y el mínimo de tres) (Cuadro 4).

En el G2, Estación Experimental Alfredo Volio Mata (EEAVM), el encargado del módulo tiene 43 años y estudios a nivel de postgrado con dieciocho años de experiencia en la actividad ganadera. La superficie es de 24 ha con una carga de 2,6 $\mathrm{UA} \mathrm{ha}^{-1}$ y una producción promedio por vaca de 14 1. El ingreso por hectárea es de USD\$3.959 con un índice tecnológico de 9 (Cuadro 4).

El G3, conformado por el productor de Cartago, presenta una edad de 56 años con estudios a nivel de bachiller y 35 años de antigüedad en la ganadería bovina. Cuenta con una superficie de 57 ha y $0,7 \mathrm{UAha}^{-1}$ y una producción promedio de 181 de leche por vaca. El ingreso por hectárea en este grupo es de 1090 y un índice tecnológico de tres. En el G4, CATIE, cuenta con una superficie de 46 ha; 4,1 $\mathrm{UAha}^{-1}$ con una producción promedio por vaca de 16,5 con un ingreso por hectárea de USD\$ 6226 y un índice tecnológico de 10.

Los productores que conformaron el G1 son productores de la provincia de Guanacaste, el G2 por la EEAVM, el G3 por el productor de Cartago y el G4 por el CATIE. La adquisición de la raza CLT Reyna

Cuadro 4. Características socioeconómicas y tecnológicas de los productores y unidades de producción con Criollo Lechero Tropical (Reyna) en las provincias de Guanacaste y Cartago, Costa Rica. 2011.

\begin{tabular}{lcccc}
\hline Variable/Tipología & G1: Guanacaste & G2: EEAMV & G3: Cartago & G4: CATIE \\
\hline Edad (años) & $57 \pm 5$ & 43 & 56 & 40 \\
Escolaridad (años) & $19 \pm 2,5$ & 25 & 15 & 20 \\
Experiencia (años) & $33 \pm 3,5$ & 18 & 35 & 12 \\
Superficie pecuaria (ha) & $60 \pm 31,6$ & 24 & 57 & 46 \\
Unidades animal (UA) & $34,6 \pm 15,2$ & 62,5 & 38 & 188 \\
Unidades animal por hectárea (UAha ${ }^{-1}$ ) & $0,6 \pm 0,1$ & 2,6 & 0,7 & 4,1 \\
Producción prom. de leche por ordeña (L) & $101 \pm 79$ & 630 & 630 & 1732,5 \\
Producción de leche por vaca (Lordeña ${ }^{-1}$ ) & $7 \pm 5$ & 14 & 18 & 16,5 \\
Ingreso (USD) & $15950 \pm 13340$ & 95004 & 62118 & 286282 \\
Ingreso por hectárea (USDha & & \\
Índice tecnológico & $297 \pm 293,75$ & 3959 & 990 & 6226 \\
\hline
\end{tabular}

G1: Productores de Guanacaste; G2: Estación Experimental Alfredo Volio Mata (EEAVM); G3: Productores de Cartago; G4: Centro Agronómico Tropical de Investigación y Enseñanza (CATIE). 
por parte de G2 y G4 fue con fines de investigación mientras que para el caso de G1 y G3 establecimiento de cruces con otras razas Bos taurus o Bos indicus.

El G1 presentó la mayor superficie pecuaria y la menor la EEAVM (Cuadro 4). Por su parte, la mayor carga animal se presentó en el G4 y el menor entre los productores de G1 . La mayor producción de leche por ordeña se presentó en el G4 y la menor en G1 mientras que la producción entre G2 y G3 fue similar.

El mayor ingreso promedio, por el número de productores que conforman este grupo, se presentó entre los productores de G1 y el menor en el G3. El mayor ingreso por hectárea se presentó en el G4, seguido de G2, G1 y G3.

A diferencia de los productores de G1 y G3, los grupos G2 y G4 no dan valor agregado al producto (leche) pero presentan mejores ingresos totales y por hectárea por el volumen de producción que manejan. El G1 presentó un índice tecnológico (IT) de 5 51,6 (máximo siete y mínimo tres) mientras que el G2, G3 y G4 presentaron un IT de nueve, tres y diez, respectivamente. Así los grupos G2 y G2 (instituciones de educación e investigación), presentaron mayor IT en comparación con el resto de los productores de G1 y G3; esto debido, posiblemente a contar con mayor presupuesto para la adquisición de tecnología.

Se encontró que los grupos con mayor IT (G2 y G4) cuentan con menor cantidad de bovinos CLT Reyna, con porcentaje de cruces con otras razas de entre el $25-50 \%$ (G2 ) y entre $50-75 \%$ (G4 ), respectivamente. La mayor cantidad de bovinos CLT Reyna se concentra en G1; no obstante, sólo una de las 7 UP cuenta con animales puros CLT Reyna.

Es importante destacar que al excluir la raza CLT Reyna, por motivos productivos y legales, tanto el G2 como en G4, estos bovinos fueron adquiridos principalmente por los productores del G1. Sin embargo, existe la posibilidad que otros productores manejen dicha raza en Costa Rica pero con la información disponible en el Ministerio de Agricultura y Ganadería (MAG), AMCROLET, y grupos encontrados (G1, G2, G3 y G4) no fue posible ubicar a más productores.

Probablemente, las instituciones de Educación e Investigación al decidir no continuar con el uso y manejo de la raza CLT Reyna, se generó aún una menor difusión e información sobre la misma, además que el Ministerio de Agricultura y Ganadería no cuenta con información alguna sobre la raza. Esto acorde a lo reportado por Hidalgo et al. (2004) y Rubio (2008) en relación a la falta de información sobre el inventario y distribución de la raza y orientación de la producción hacia la exportación de semen, principalmente.

Los entrevistados manifestaron que dentro de las principales fortalezas de la raza CLT Reyna están la rusticidad, adaptación al medio, resistencia a parásitos y enfermedades, posibilidad de alimentación exclusiva a base de pastos, mansedumbre y longevidad. Contrariamente, las debilidades, según los entrevistados, son consanguinidad, mala conformación corporal y periodos cortos de lactancia.

La consanguinidad, puede deberse a que la mayoría de los bovinos y semen adquiridos procedieron de un mismo hato (Rivas, Nicaragua); posteriormente G2 y G4 los distribuyeron entre los productores y éstos entre ellos mismos.

Es importante destacar que los entrevistados manifestaron que es recomendable el uso de la raza CLT Reyna para pequeños y medianos productores como una alternativa de producción de leche en el trópico; no obstante, mencionaron que dicha raza sólo debe utilizarse mediante cruces con razas especializadas como Holstein y Jersey; según los entrevistados, razas base para la producción de leche y mediante las cuales se pueden establecer diversos cruces. Finalmente hubo un consenso, entre los entrevistados, en que hace falta mayor difusión sobre la CLT Reyna así como esquemas de apoyo, mejoramiento genético e información sobre el potencial de ésta para la producción de leche en el trópico.

En Costa Rica, el uso y manejo de la raza CLT Reyna ha ido en detrimento debido a políticas institucionales, aspectos legales y productivos además de haber poca información y difusión de ésta entre los productores así como en relación al inventario y distribución de la misma. Por ello, la raza ha dejado de tener importancia en los sistemas ganaderos costarricenses productores de leche. Se encontraron 81 bovinos Reyna, en diversos porcentajes de cruces con otras razas Bos taurus x Bos indicus, distribuidos en siete unidades de producción (UP) en las provincias de Cartago y Guanacaste. Sólo una de estas siete UP cuenta con bovinos puros. Los cruces con CLT Reyna se utilizan principalmente para obtener adaptabilidad y resistencia al medio.

Mediante el análisis cluster se identificaron cuatros grupos que utilizan la raza CLT Reyna; de estos 
dos son instituciones (29\%) que han decidido desechar el uso de la raza y cinco productores independientes (71\%) que en mayor o menor grado continúan con el manejo de la misma.

\section{AGRADECIMIENTOS}

Al Food Security Center (FSC) de la Universidad Hohenheim, Alemania por la beca otorgada para la realización de la pasantía postdoctoral. A los entrevistados por su apertura, disposición y participación en las entrevistas, aportando en algunos casos información sensible.

\section{LITERATURA CITADA}

Alfaro, O; Ducca, E; Solano, OM; Zumbado, C. 2007. Ministerio de Agricultura (MAG). Caracterización y plan de acción para el desarrollo de la agrocadena de ganado bovino en la región de Huetar Norte. 65 p. (en línea). Disponible en http://www.mag.go.cr/bibliotecavirtual/ac-ganaderia-rhn-2007.pdf.

Asociación Mexicana de Criadores de Ganado Romosinuano Y Criollo Lechero TropicaL). 1998. Reglamento técnico de la Asociación Mexicana de Criadores de ganado Romosinuano y Lechero tropical. AMCROLET. 12 p.

AMCROLET (Asociación Mexicana de Criadores de Ganado Romosinuano y Criollo Lechero Tropical). 2010. Archivo inventario bovino octubre 2010. AMCROLET. Veracruz, México. 34 p.

Atlas Cantonal de Costa Rica. IFAM. 1987. 396 p. (en línea). Consultado marzo 2011 Disponible en http://www. sitiosdecostarica.com/indexCantones.htm

Casas, E; Tewolde, A. 2001. Evaluación de características relacionadas con la eficiencia reproductiva de genotipos criollos lecheros en el trópico húmedo. Arch. Latinoam. Prod. Anim. 9(29):63-67.

Cervantes, P; Fernández, L; Ponce, P. 2006. Caracterización de las curvas de lactancia en producción y componentes mayores de la leche en diferentes razas y cruzas en las condiciones del trópico mexicano. Rev. Salud Anim. 28(2):90-95.

Cámara Nacional de Productores de Leche. 2004. Información del sector ganadero Año 2004. (en línea). Consultado marzo 2011. Disponible en http://www. proleche.com/info_sector.htm
Corporación Ganadera (Corfoga). Censo ganadero 2000. (en línea). Disponible en http://www.corfoga.org/ images/public/documentos/pdf/otros_cuadros.pdf. Marzo de 2011.

Deaton, O. 1979. El mejoramiento genético del ganado criollo lechero tropical. Seminario tópicos de investigación en mejoramiento animal. San Salvador. 15 p.

De Alba, J. 2007. El Ganado lechero tropical en América Latina. Departamento de Agricultura. FAO. 3 p. (en línea). Consultado diciembre 2010. Disponible en www.produccion-animal.com.ar

De Alba, J. 2011. Los criollos lecheros tropicales. In El libro de los bovinos criollos de América, J. de Alba Martínez. Biblioteca Básica de Agricultura (Colegio de Postgraduados), Ediciones Papiro Omega S.A. de C.V. p. 92-98.

Díaz, P; Alba, J; Becerril, C; Rosendo, A; Guerrero, J; Estrella, A; Rueda, B. 2005. El ganado Crilet, una alternativa viable para la producción de leche en el trópico mexicano. Colegio de Postgraduados Campus Veracruz. (en línea). Consultado diciembre 2010. Disponible en www.colpos.mx/cveracruz/SubMenu_ Publi/pdf/CRILET.pdf

Estrella, G; Cervantes, P; Cisneros, N; Becerril, C; Pérez, P; Rosendo, A. 2004. Características fisicoquímicas del yogurt elaborado con leche de vacas Criollo Lechero Tropical. Colegio de Postgraduados. (en línea). Consultado diciembre 2010. Disponible en http://www. colpos.mx/cveracruz/SubMenu_Publi/Avances2004/ caracterizacion_yogurth_criollo.html

González, F; Becerril, C; Pérez Torres, G; Díaz, P. 2009. Ticks infesting body regions of tropical milking criollo cattle in Veracruz, Mexico. Agrociencia 43:11-19.

Guerrero, J; Cervantes, P; Cisneros, N; Becerril, C; Pérez, P; Rosendo, A. 2004. Composición láctea y rendimiento quesero de vacas de la raza Criollo Lechero Tropical. Colegio de Postgraduados Campus Veracruz. 6 p. (en línea). Disponible en http://www.colpos.mx/ cveracruz/SubMenu_Publi/Avances2004/queso_crio1lo.html

Hernández, A; Cervantes, P; Salinas, V; García, R; Tejeda, A; Gallardo, F; Álvarez, J. 2007. Respuesta al estrés por calor en la vaca criollo lechero tropical bajo un sistema de doble propósito en México. Rev. Salud. Anim. 29(2):85-90.

Hidalgo, C; Monge, Cruz, A; Molina, Jr; Camacho, J; Vargas, G; Barrientos, O. 2004. Informe parcial del país sobre la situación nacional de los recursos zoo 
genéticos. Subcomisión nacional encargada de la elaboración del informe país sobre la situación de los recursos genéticos pecuarios de Costa Rica. 43 p.

Magofke, JC. 1964. Estimación del mejoramiento genético en producción de leche, grasa y largo de lactancia en el ganado Criollo Lechero de Turrialba. OEA. Costa Rica. 110 p.

Martínez, J; Aguirre, J; Martínez, G; Torres, G. 2006. Comportamiento productivo y reproductivo de tres genotipos bovinos en la región del Soconusco, Chiapas, México. Zootecnia Tropical 24(2):109-120.

MAG (Ministerio de Agricultura y Ganadería). 2007. Agrocadena de leche año 2007. Dirección Central Regional Oriental. Costa Rica. 109 p. (en línea). Consultado marzo 2011. Disponible en http://www.mag.go.cr/ bibliotecavirtual/a00079.pdf

Müller-Hayer, B; Gelman, J. 1981. Recursos genéticos animales en América Latina. Ed. Müller-Hayer, Berndt y Gelman, Juan. Departamento de Agricultura, FAO. Roma. 315 p.

Pariacote, F. 2000. Riesgos de extinción del conglomerado nativo de genes bovinos en América Latina: Caso Venezuela. Archivos Zootecnia 49:17-26.

Primo, AT. 1992. El ganado bovino ibérico en las Américas: 500 años después. Archivos de Zootecnia 41 (extra): 421-432.

Rosendo, A; Becerril, C. 2002. Productive performance and genetic parameters in the tropical milking Criollo Cattle in Mexico. Proc. 7th. World Congress on Genetics Applied to Livestock Production. Montpellier, France. Communication 25-25 p.
Rouse, JE. 1977. The criollo. Spanish cattle in the Americas. University of Oklahoma Press. Oklahoma. 303 p.

Rubio, A. 2008. Caracterización técnica y económica de la raza de ganado Reina en la Finca El Pino, Rivas, Nicaragua. Tesis. Escuela Agrícola Panamericana, El Zamorano, Honduras. 24 p. (en línea). Disponible en http://zamo-oti02.zamorano.edu/tesis_infolib/2008/ T2657.pdf. 2008.

Santellano, E; Becerril, C; Yu Mei-C; Gianola, D; Torres, G; Ramírez, R; Domínguez, J; Rosendo, A. 2011. Caracterización de la lactancia y evaluación genética del ganado Criollo Lechero Tropical utilizando un modelo de regresión aleatoria. Agrociencia 45(2):165175.

SEPSA (Secretaría Ejecutiva de Planificación Sectorial Agropecuaria). 2002. Desempeño de la ganadería de leche y de la industria de la transformación de productos lácteos en Costa Rica 1996 - 2001. 32 p

Wilkins, V. 1991. Criollo Cattle of the America. Centro de Investigación Agrícola Tropical. Santa Cruz, Bolivia. 19 p. (en línea). Consultado mayo 2011. Disponible en http://www.fao.biodiversity/idad

Vaughan, B; Rojas, E; Quesada, L. 2005. Diálogo sobre recursos genéticos animales en la agricultura: la necesidad de su conservación y mejora para lograr sistemas de producción competitivos en Mesoamérica. Memoria. Montelimar, Nicaragua. p. 107-124.

Vilaboa, J; Díaz, P. 2009. Caracterización socioeconómica y tecnológica de los sistemas ganaderos en siete municipios del estado de Veracruz, México. Zootecnia Trop. 27(4):427-436. 